\title{
A NOTE ON LONGITUDINAL FIELDS IN THE WEYL EXPANSION OF THE ELECTROMAGNETIC GREEN TENSOR
}

\author{
GREGOR PIEPLOW, HARALD R. HAAKH and CARSTEN HENKEL \\ Institut für Physik und Astronomie, Universität Potsdam, \\ Karl-Liebknecht-Str. 24/25, 14476 Potsdam, Germany \\ pieplow@uni-potsdam.de
}

Published 28 July 2012

\begin{abstract}
Many calculations of dispersion interactions between atoms and macroscopic bodies or between two bodies make use of Green tensors. The expansion of this tensor in polarizations allows for an anatomic interpretation of the interaction. In planar systems with partial translation invariance, the Weyl representation of the Green tensor is often applied. Although it is transverse in this representation, we argue that the field it describes contains nonradiative parts as well. This can be seen by calculating the Green tensor in the momentum representation and observing certain cancellations among longitudinal and transverse contributions.
\end{abstract}

Keywords: Green tensor; Polarization; Quantum friction on a moving atom.

PACS numbers: 78.20.Ci, 03.50.De, 68.35.Af

\section{Introduction}

The force exerted on a small neutral particle in front of a half space is one of the basic problems encountered in the research on dispersion forces. and has been considered as early as 1932 by Lennard-Jones ${ }^{1}$. Casimir and Polder ${ }^{2}$ connected the atom-surface interaction for a particle in rest to the fluctuations of the electromagnetic vacuum field and technological progress in recent years has made it feasible to measure Casimir-Polder forces with great precision ${ }^{3}$. While the theoretical description of the atom-wall interaction for a particle at rest is well-established ${ }^{4,5,6}$, the case of bodies in relative motion has been controversially discussed ${ }^{7,8,9}$. To the day, it is not clear whether a particle is subject to a force component opposing its motion, i.e. to van der Waals or Casimir friction. Many strategies employ employ two point correlation tensors, namely Green tensors, to calculate these forces in a first order perturbation theory. The question of analyzing these tensors with respect to polarizations arose studying quantum friction. A detailed physical interpretation was sought in order to compare the different approaches. There is a number of different approaches to this problem in the literature, and it is desirable to have a clear interpretation 
of the underlying description to be able to compare the results. An analysis of two-point correlation functions of the electromagnetic field and the related Green tensors is of advantage because they are response functions and their regularization is often straightforward. In the general case, both longitudinal and transverse field components occur. The longitudinal part of an electromagnetic field is closely related to the Coulomb potential due to the charge density, while the transverse part of a field is commonly associated with radiation. However, distinguishing the parts of the fields that mediate the interaction can become quite tedious when Green tensors are used. We will show that the appearance of these tensors can be misleading as in the case of the Weyl representation, where the field components are decomposed in a basis adapted to a system with a planar symmetry. The tensor is transverse, which can be misleading because it in fact connects longitudinal as well as transverse currents to the respective fields. In the following, we illustrate this issue by carefully constructing the tensor in the momentum representation, starting from the Green tensor in free space. This allows keeping track of all the polarizations. Calculating the Weyl representation will allow a comparison with the expressions found in the literature.

\section{Green tensor above a medium half-space}

\subsection{Gauge-invariant definition}

The starting point of our analysis is the retarded free space Green tensor associated with the Maxwell equations in the momentum representation. In Gauss units, the well-known current-field relation reads

$$
\left[-\mathbf{k} \otimes \mathbf{k}+\mathbb{1}\left(\mathbf{k}^{2}-\frac{\omega^{2}}{c^{2}}\right)\right] \mathbf{E}=4 \pi \frac{i \omega}{c} \mathbf{j} .
$$

The Green tensor is defined through the field created by a point-current

$$
\mathbf{E}(\omega, \mathbf{k})=\mathcal{G}(\omega, \mathbf{k}) \cdot \mathbf{j}(\omega, \mathbf{k}),
$$

which gives the retarded free space Green tensor

$$
\mathcal{G}_{\text {free }}(\omega, \mathbf{k})=\frac{4 \pi i \omega / c}{\mathbf{k}^{2}-\omega^{2} / c^{2}}\left(\mathbb{1}-\frac{c^{2}}{\omega^{2}} \mathbf{k} \otimes \mathbf{k}\right),
$$

where the frequency $\omega$ is kept in the upper half-plane to account for the retardation of the tensor. Note that this definition is based on the fields only and does not require a gauge condition. Neither are there constraints on the current density regarding its longitudinal or transverse part. This version of the Green tensor can now be used to compute the tensor for two points above a medium-filled half space.

\subsection{Weyl representation in free space}

The calculation of the Weyl representation of the free space Green tensor will provide some insight into the more complicated situation, where a half space breaks the 


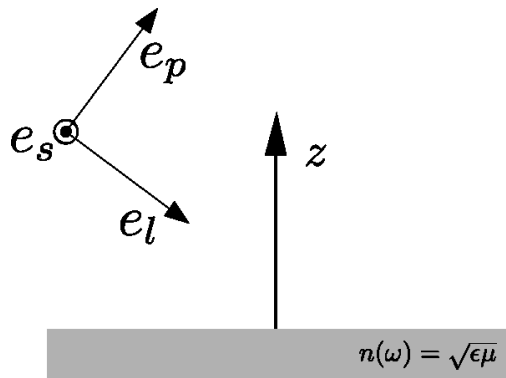

Fig. 1. Polarization basis for an incident wave. The surface normal $\mathbf{n}$ and the wave-vector $\mathbf{k}=k \mathbf{e}_{k}$ span the plane of incidence. The electric field component in $p$-polarization is parallel to this plane, while a $s$-polarized field is oriented perpendicularly.

symmetry. We start by decomposing $\mathcal{G}(\omega, \mathbf{k})$ into orthogonal polarizations adapted to the plane of incidence spanned by $\mathbf{k}$ and the surface normal $\mathbf{e}_{z}$ (see Fig.1).

$$
\mathbf{e}_{p}=\frac{1}{k q}\left(-k_{z} k_{x},-k_{z} k_{y}, q^{2}\right), \mathbf{e}_{s}=\frac{1}{q}\left(-k_{y}, k_{x}, 0\right), \mathbf{e}_{k}=\frac{1}{k}\left(k_{x}, k_{y}, k_{z}\right),
$$

where $q=\left(k_{x}^{2}+k_{y}^{2}\right)^{1 / 2}$. The orthonormal vectors $\mathbf{e}_{p}, \mathbf{e}_{s}, \mathbf{e}_{k}$ describe, respectively, orientations in the plane of incidence and perpendicular to it, where the vector $\mathbf{e}_{k}$ is parallel to the incident wave vector. The free tensor can then be decomposed as:

$$
\mathcal{G}_{\text {free }}(\omega, \mathbf{k})=\frac{4 \pi i \omega / c}{k^{2}-\omega^{2} / c^{2}}\left(\mathbf{e}_{p} \otimes \mathbf{e}_{p}+\mathbf{e}_{s} \otimes \mathbf{e}_{s}\right)-4 \pi i \frac{c}{\omega} \mathbf{e}_{k} \otimes \mathbf{e}_{k} .
$$

The planar symmetry suggests a mixed momentum-space representation, obtained from the Fourier integral with respect to $k_{z}$

$$
\mathcal{G}\left(\omega, \mathbf{q} ; z, z^{\prime}\right)=\int_{-\infty}^{\infty} d k_{z} \mathcal{G}_{\text {free }}(\omega, \mathbf{k}) e^{i k_{z}\left(z-z^{\prime}\right)},
$$

where $\mathbf{q}=\left(k_{x}, k_{z}\right)$ is a two dimensional in-plane wave vector. The integration is performed with contour techniques, using the analytical continuation of the integrand into the complex $k_{z}$-plane. We begin by analyzing the tensor's pole structure. The scalar propagator $1 /\left(k^{2}-\omega^{2} / c^{2}\right)$ has poles at $k_{z}= \pm \kappa$, where

$$
\kappa=\left\{\begin{array}{ll}
\sqrt{\omega^{2} / c^{2}-q^{2}}, & q^{2}<\operatorname{Re} \omega^{2} / c^{2} \\
i \sqrt{q^{2}-\omega^{2} / c^{2}}, & q^{2}>\operatorname{Re} \omega^{2} / c^{2}
\end{array} .\right.
$$

Additional poles at $k_{z}= \pm i q$ arise from the factor $1 / k$ in the polarization vectors $\mathbf{e}_{p}$ and $\mathbf{e}_{k}$ [Eq. (4)]. For $z>z^{\prime}$, the integration contour can be closed in the upper half-plane, and we are left with the residues there. The residues of $\mathbf{e}_{k}$ and $\mathbf{e}_{p}$ are not independent, as the following calculation at the pole $k_{z}=i q$ shows

$$
A=\operatorname{Res}\left[\frac{1}{k^{2}-\omega^{2} / c^{2}} \mathbf{e}_{p} \otimes \mathbf{e}_{p}, k_{z}=i q\right] \text {. }
$$




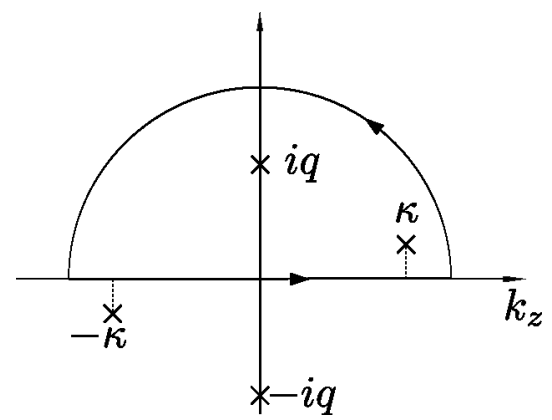

Fig. 2. Integration contour for $z>z^{\prime}$. For $z<z^{\prime}$, the contour is closed in the lower $k_{z}$-plane, due to the factor $e^{i k_{z}\left(z-z^{\prime}\right)}$ in Eq. (6).

The denominator becomes $-c^{2} / \omega^{2}$. In the polarization projector, we rewrite

$$
\frac{1}{q^{2} k^{2}}=\frac{1}{q^{2}}\left(\frac{1}{2 i q} \frac{1}{k_{z}-i q}-\frac{1}{2 i q} \frac{1}{k_{z}+i q}\right) .
$$

Hence the residue is

$$
A=\frac{c^{2}}{2 i q \omega^{2}}\left(\begin{array}{l}
k_{x} \\
k_{y} \\
i q
\end{array}\right)\left(k_{x}, k_{y}, i q\right)=\operatorname{Res}\left[\frac{c^{2}}{\omega^{2}} \mathbf{e}_{k} \otimes \mathbf{e}_{k}, k_{z}=i q\right] .
$$

Due to the last equality, the longitudinal projector $\mathbf{e}_{k} \otimes \mathbf{e}_{k}$ cancels and does not appear in the Weyl expansion. Collecting all the residues and considering also the case $z<z^{\prime}$, the complete tensor takes the form

$$
\mathcal{G}\left(\omega, \mathbf{q} ; z, z^{\prime}\right)=\frac{2 \pi i \omega}{c \kappa} e^{i \kappa\left|z-z^{\prime}\right|}\left[\mathbf{e}_{s} \otimes \mathbf{e}_{s}+\mathbf{e}_{p} \otimes \mathbf{e}_{p}\right]_{k_{z}=\kappa \operatorname{sign}\left(z-z^{\prime}\right)} .
$$

This is a well-known result ${ }^{10}$.

\subsection{Source and field above a planar interface}

\subsubsection{Scattering problem}

We look for a Green tensor that connects a current source above a plane surface to the field reflected from it. We start with an interpretation of

$$
\mathbf{E}(\mathbf{x}, t)=\int_{-\infty}^{\infty} d^{3} k d \omega \mathcal{G}_{\text {free }}(\omega, \mathbf{k}) \cdot \mathbf{j}(\omega, \mathbf{k}) e^{i\left(\mathbf{k} \cdot\left(\mathbf{x}-\mathbf{x}^{\prime}\right)-\omega t\right)}
$$

The phase $-\mathbf{k} \cdot \mathbf{x}^{\prime}$ corresponds a shift of the current distribution to a position $\mathbf{x}^{\prime}$ in the upper half space. This equation motivates an ansatz for the field created by a point-like current above a half space in terms of a scattering problem: the term $\mathcal{G}_{\text {free }}(\omega, \mathbf{k}) \cdot \mathbf{j}(\omega, \mathbf{k}) e^{i \mathbf{k} \cdot\left(\mathbf{x}-\mathbf{x}^{\prime}\right)}$ under the integral is interpreted as a wave incident on 
the surface. The standard scattering approach therefore requires a field of the form

$$
\mathbf{E}(z)=\left\{\begin{array}{lc}
\mathcal{G}_{\text {free }}(\omega, \mathbf{k}) \cdot \mathbf{j}(\omega, \mathbf{k}) e^{-i k_{z}\left(z-z^{\prime}\right)}+\mathbf{E}^{\mathrm{out}}(\omega, \mathcal{M} \cdot \mathbf{k}) e^{i k_{z}\left(z+z^{\prime}\right)}, & z>0 \\
\mathbf{E}^{\operatorname{tr}}\left(\omega, \mathbf{k}^{\prime}\right) e^{-i k_{z}^{\prime}\left(z-z^{\prime}\right)}, & z<0
\end{array}\right.
$$

where the matrix $\mathcal{M}=\operatorname{diag}(1,1,-1)$ implements the mirror reflection at the interface. The $\mathbf{q}$ dependence can be suppressed, since this wave vector is conserved. We decompose the fields into the polarization basis [Eq. (4)], see Fig. 1.

The reflected $\left(\mathbf{E}^{\text {out }}\right)$ and transmitted $\left(\mathbf{E}^{\text {tr }}\right)$ fields are found by matching at the interface. We work out here the case where the medium in the half-space $z<0$ is described by macroscopic boundary conditions ${ }^{12}$. The reflection laws for the $s$ and $p$-polarization are well-known. The reflection coefficient $r_{k}$ for the longitudinal polarization which does not usually appear in this context, can be found for example in Ref. 13, where a generalized Coulomb gauge was used. The same result emerges from the boundary conditions for a strictly longitudinal field (scalar potential). We use for the reflected field the basis vectors

$$
\mathbf{e}_{s}^{r}=\mathcal{M} \cdot \mathbf{e}_{s}=\mathbf{e}_{s}, \mathbf{e}_{p}^{r}=-\mathcal{M} \cdot \mathbf{e}_{p}, \mathbf{e}_{k}^{r}=\mathcal{M} \cdot \mathbf{e}_{k},
$$

This fixes the signs of the reflection coefficients:

$$
r_{s}=\frac{k_{z}-k_{z}^{\prime}}{k_{z}+k_{z}^{\prime}}, \quad r_{p}=\frac{n^{2} k_{z}-k_{z}^{\prime}}{n^{2} k_{z}+k_{z}^{\prime}}, \quad r_{k}=\frac{k_{z}-n^{2} k_{z}^{\prime}}{k_{z}+n^{2} k_{z}^{\prime}} .
$$

where $n=n(\omega)$ is the refractive index of the medium and $k_{z}^{\prime}=\left[n^{2} k_{z}^{2}+\left(n^{2}-\right.\right.$ 1) $\left.\left(k_{x}^{2}+k_{y}^{2}\right)\right]^{1 / 2}$. We construct a reflection matrix that relates the incident field with the outgoing field (basically the S-matrix for this type of geometry)

$$
\begin{aligned}
\mathbf{E}^{\text {out }}(\omega, \mathcal{M} \cdot \mathbf{k}) & =\mathcal{R}(\omega, \mathbf{k}) \cdot \mathbf{E}^{\text {in }}(\omega, \mathbf{k}) \\
& =\mathcal{M} \cdot\left(r_{s} \mathbf{e}_{s} \otimes \mathbf{e}_{s}-r_{p} \mathbf{e}_{p} \otimes \mathbf{e}_{p}+r_{k} \mathbf{e}_{k} \otimes \mathbf{e}_{k}\right) \cdot \mathbf{E}^{\text {in }}(\omega, \mathbf{k}),
\end{aligned}
$$

where the field amplitude incident on the surface is given by

$$
\mathbf{E}^{\mathrm{in}}(\omega, \mathbf{k})=\mathcal{G}_{\text {free }}(\omega, \mathbf{k}) \cdot \mathbf{j}(\omega, \mathbf{k}) e^{-i k_{z} z^{\prime}} .
$$

Eq. (16) provides the field that is present in addition to the free-space radiation, due to the boundary conditions at the surface. This is our starting point for finding the Weyl representation of the Green tensor above the surface.

\subsubsection{Weyl representation}

We can now read off the Green tensor above the surface by combining Eqs. (13) and (16) and writing the result in a form analogous to Eq. (12)

$$
\mathbf{E}(\mathbf{x}, t)=\int_{-\infty}^{\infty} d^{3} k d \omega \mathcal{G}(\omega, \mathbf{k}) \cdot \mathbf{j}(\omega, \mathbf{k}) e^{i\left(\mathbf{k} \cdot\left(\mathbf{x}-\mathbf{x}^{\prime}\right)-\omega t\right)}
$$

We find for $z, z^{\prime}>0$

$$
\mathcal{G}(\omega, \mathbf{k})=\mathcal{G}_{\text {free }}(\omega, \mathbf{k})+\mathcal{R}(\omega, \mathbf{k}) \cdot \mathcal{G}_{\text {free }}(\omega, \mathbf{k}) e^{2 i k_{z} z^{\prime}}
$$


Now we can calculate the Weyl representation of the reflected part

$$
\mathcal{G}^{\mathrm{R}}\left(\omega, \mathbf{q} ; z, z^{\prime}\right)=\frac{1}{2 \pi} \int_{-\infty}^{\infty} d k_{z} \mathcal{R}(\omega, \mathbf{k}) \cdot \mathcal{G}_{\text {free }}(\omega, \mathbf{k}) e^{2 i k_{z} z^{\prime}} e^{i k_{z}\left(z-z^{\prime}\right)} .
$$

The integration is again performed by analytical continuation of $\mathcal{G}$ into the complex $k_{z}$ plane, using the contour shown Fig. 3. We obtain the pole structure from

$$
\begin{aligned}
-i \frac{c}{4 \pi \omega} \mathcal{R}(\omega, \mathbf{k}) \cdot \mathcal{G}_{\text {free }}(\omega, \mathbf{k})= & \frac{1}{k^{2}-\omega^{2} / c^{2}} r_{s} \mathbf{e}_{s} \otimes \mathbf{e}_{s} \\
& -\frac{1}{k^{2}-\omega^{2} / c^{2}} r_{p} \mathcal{M} \cdot \mathbf{e}_{p} \otimes \mathbf{e}_{p}-\frac{c^{2}}{\omega^{2}} r_{k} \mathcal{M} \cdot \mathbf{e}_{k} \otimes \mathbf{e}_{k} .
\end{aligned}
$$

It turns out that the reflection coefficients do not contribute with any poles. When considering for example $r_{p}$, one finds that the surface plasmon dispersion relation does not give singularities in the upper half-plane if the root for the medium wavevector $k_{z}^{\prime}$ is cut along the negative $k_{z}$ axis. The poles at $k_{z}= \pm \kappa$ [Eq. (7)] are again due to the scalar propagator in Eq. (21) and induce the usual dispersion relation between $k$ and $\omega$. At the pole $k_{z}=i q$ due to the polarization vectors, a cancellation similar to the free-space Weyl expansion [Eq.(10)] occurs also in the reflection coefficients

$$
\begin{aligned}
k_{z} \rightarrow i q: \quad r_{p} & =\frac{k_{z}-n^{2} k_{z}^{\prime}}{k_{z}+n^{2} k_{z}^{\prime}} \rightarrow \frac{1-n^{2}}{1+n^{2}}, \\
r_{k} & =\frac{n^{2} k_{z}-k_{z}^{\prime}}{n^{2} k_{z}+k_{z}^{\prime}} \rightarrow \frac{n^{2}-1}{n^{2}+1},
\end{aligned}
$$

and the residues for the reflected projectors are [compare with Eq. (10)]

$$
\operatorname{Res}\left[\frac{1}{k^{2}-\omega^{2} / c^{2}} \mathcal{M} \cdot \mathbf{e}_{p} \otimes \mathbf{e}_{p}, k_{z}=i q\right]=\operatorname{Res}\left[\frac{1}{\omega^{2} / c^{2}} \mathcal{M} \cdot \mathbf{e}_{k} \otimes \mathbf{e}_{k}, k_{z}=i q\right] \text {. }
$$

So again, the contribution from the longitudinal polarization is cancelled by a part from the $p$-polarization. Finally, the Weyl representation of the reflected part of the

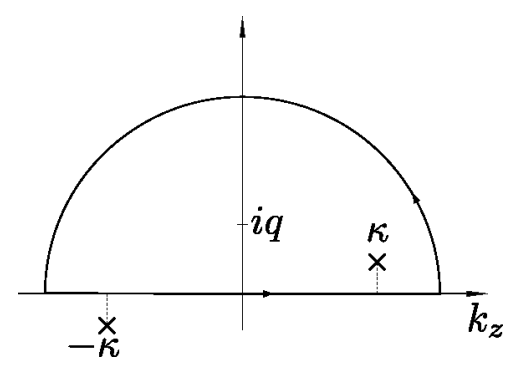

Fig. 3. Integration contour for Eq. (20) in the complex $k_{z}$-plane. 
Green tensor becomes

$$
\mathcal{G}^{\mathrm{R}}\left(\omega, \mathbf{q} ; z, z^{\prime}\right)=-\frac{2 \pi \omega}{c \kappa} e^{i \kappa\left(z+z^{\prime}\right)}\left[r_{s} \mathbf{e}_{s} \otimes \mathbf{e}_{s}-r_{p} \mathcal{M} \cdot \mathbf{e}_{p} \otimes \mathbf{e}_{p}\right]_{k_{z}=\kappa}
$$

This is the expression found in textbooks and the literature, see e.g. Ref.14.

\section{Conclusion}

The Weyl representation of the Green tensor above a dielectric half space has been calculated by means of a decomposition of the free space Green tensor in momentum representation and imposing the usual continuity conditions at the boundary, without fixing a gauge or putting further constraints on the fields or the current density. A longitudinal field component appears, which is unusual in this context. The Green tensor found this way is consistent with the Green tensor usually found in the literature. Although the tensor is transverse in the Weyl representation it contains nonradiative contributions.

\section{References}

1. J. E. Lennard-Jones Trans. Faraday Soc. 28 333-359 (1932)

2. H. B. G. Casimir, D. Polder, Phys. Rev. 73360 (1948)

3. J. M. Obrecht, R. J. Wild, M. Antezza, L. P. Pitaevskii, S. Stringari, E. A. Cornell Phys. Rev. Lett. 9863201 (2007)

4. S. Scheel, S. Y. Buhmann, Acta Physica Slovaca 58 675-809 (2008)

5. J. M. Wylie and J. E. Sipe, Phys. Rev. A 322080 (1985)

6. V. Bezerra, G. Klimchitskaya, V Mostepanenko, C. Romero, Phys. Rev. A 78, 042901 (2008)

7. J. B. Pendry J. Phys. C 910301 (1997)

8. T. G. Philbin and U. Leonhardt New J. Phys. 11033035 (2009)

9. G. Dedkov and A. Kyasov A., Phys. Sol. St. 51 1-26 (2009)

10. L. D. Landau, E.M. Lifshitz and L. P. Pitaevskii, Statistical Physics Part 2, (Heinemann, Oxford, 1980)

11. C. Tannoudji, J. Dupont-Roc and G. Grynberg, Photons and Atoms: Introduction to Quantum Electrodynamics, 1st edn. (Wiley, 1997, New York)

12. J. D. Jackson Classical Electrodynamics, 3rd edn. (Wiley, 1998, New York)

13. C. Eberlein and D. Robaschik, Phys. Rev. D 73025009 (2006)

14. J. E. Sipe, J. Opt. Soc. Am. B 4, 481 (1987) 